\title{
RESEARCH
}

Open Access

\section{Psychometric characterization of incidental feedback sources during grasping with a hand prosthesis}

\author{
Meike Annika Wilke ${ }^{1,2^{*}}$ (D), Christian Niethammer ${ }^{2,3}$, Britta Meyer ${ }^{2}$, Dario Farina ${ }^{2,4}$ and Strahinja Dosen ${ }^{2,5}$
}

\begin{abstract}
Background: A prosthetic system should ideally reinstate the bidirectional communication between the user's brain and its end effector by restoring both motor and sensory functions lost after an amputation. However, current commercial prostheses generally do not incorporate somatosensory feedback. Even without explicit feedback, grasping using a prosthesis partly relies on sensory information. Indeed, the prosthesis operation is characterized by visual and sound cues that could be exploited by the user to estimate the prosthesis state. However, the quality of this incidental feedback has not been objectively evaluated.

Methods: In this study, the psychometric properties of the auditory and visual feedback of prosthesis motion were assessed and compared to that of a vibro-tactile interface. Twelve able-bodied subjects passively observed prosthesis closing and grasping an object, and they were asked to discriminate (experiment I) or estimate (experiment II) the closing velocity of the prosthesis using visual (VIS), acoustic (SND), or combined (VIS + SND) feedback. In experiment II, the subjects performed the task also with a vibrotactile stimulus $(V I B)$ delivered using a single tactor. The outcome measures for the discrimination and estimation experiments were just noticeable difference (JND) and median absolute estimation error (MAE), respectively.
\end{abstract}

Results: The results demonstrated that the incidental sources provided a remarkably good discrimination and estimation of the closing velocity, significantly outperforming the vibrotactile feedback. Using incidental sources, the subjects could discriminate almost the minimum possible increment/decrement in velocity that could be commanded to the prosthesis (median JND $<2 \%$ for SND and VIS + SND). Similarly, the median MAE in estimating the prosthesis velocity randomly commanded from the full working range was also low, i.e., approximately 5\% in SND and VIS + SND.

Conclusions: Since the closing velocity is proportional to grasping force in state-of-the-art myoelectric prostheses, the results of the present study imply that the incidental feedback, when available, could be usefully exploited for grasping force control. Therefore, the impact of incidental feedback needs to be considered when designing a feedback interface in prosthetics, especially since the quality of estimation using supplemental sources (e.g., vibration) can be worse compared to that of the intrinsic cues.

Keywords: Myoelectric prostheses, Upper limb, Artificial sensory feedback, Incidental feedback, Vibrotactile stimulation

\footnotetext{
* Correspondence: meikeannika.schweisfurth@haw-hamburg.de

'Department of Biotechnology, University for Applied Sciences Hamburg, Hamburg, Germany

${ }^{2}$ Advanced Rehabilitation Technology (ART) Lab, Department for Trauma Surgery, Orthopaedics and Plastic Surgery, Universitätsmedizin Göttingen (UMG), Göttingen, Germany

Full list of author information is available at the end of the article
}

C The Author(s). 2019 Open Access This article is distributed under the terms of the Creative Commons Attribution 4.0 International License (http://creativecommons.org/licenses/by/4.0/), which permits unrestricted use, distribution, and reproduction in any medium, provided you give appropriate credit to the original author(s) and the source, provide a link to the Creative Commons license, and indicate if changes were made. The Creative Commons Public Domain Dedication waiver (http://creativecommons.org/publicdomain/zero/1.0/) applies to the data made available in this article, unless otherwise stated. 


\section{Background}

Humans can effortlessly grasp and manipulate objects of very different properties, from heavy and robust to delicate and fragile. This is possible thanks to a sophisticated musculoskeletal structure innervated by a network of sensorimotor nerves, providing advanced motor commands and a comprehensive multimodal feedback (e.g., touch, proprioception, force). The studies in human motor control demonstrate that the somatosensory feedback is indeed instrumental for the planning and execution of grasping [1-3].

After an amputation of the hand, the motor and sensory functions are lost. The lost motor functions can be restored to a certain degree using myoelectric prostheses. These systems are controlled by recording the electrical activity of the user's muscles to estimate the motion intention, which is then translated into prosthesis commands [4]. Typically, the wrist and hand flexor and extensor muscles are used to command prosthesis closing and opening proportionally, thereby resulting in an intuitive connection between the user's brain and the artificial device [5]. However, current prosthetic systems are controlled in open loop, without explicitly providing any somatosensory feedback on the prosthesis state (e.g., hand aperture or grasping force) to the user. In order to truly compensate for the missing biological limb, it is commonly assumed that a prosthetic system needs to establish a bilateral communication to the user's brain, by restoring both motor and sensory functions [6, 7].

Intuitive methods to provide somatosensory feedback have been a research topic for several decades [8], and substantial progress was made in recent years [9-12]. However, a commercially available solution capable of improving the prosthesis performance in the activities of daily living is still unavailable $[13,14]$. There is a single commercial prosthesis (VINCENTevolution 2, Vincent Systems, DE) equipped with a simple vibratory feedback on the grasping force, but its clinical and functional utility has not been yet demonstrated. From the technical viewpoint, the non-invasive artificial feedback can be implemented using relatively simple solutions. A common approach is to use sensory substitution, where the lost sensory information is transmitted using alternative sensory modalities that are still spared following the amputation [15]. To this aim, the prosthesis is equipped with force and position sensors, and the sensor data are transmitted to the user by stimulating the skin of the residual limb to activate the tactile sense. The stimulation can be delivered using low-intensity electrical pulses [16] or vibration motors [17], and the information is conveyed by modulating the stimulation parameters. For example, vibration intensity and/or frequency can be proportionally associated to hand aperture [18] or grasping force [18-21]. In addition, the feedback can be provided using direct mechanical stimulation through skin stretch [22], movement on the skin [23], pressure cuffs/braces [24], or linear pushers [25-27]. More recently, several invasive solutions that directly stimulate the peripheral nerves [28-33] or somatosensory areas of the brain [34] have been presented.

Despite several methods have been successfully implemented and tested for restoring sensory feedback, the actual benefits of the artificial feedback are still elusive. Some studies showed significant improvements in performance with artificial somatosensory feedback [35-37]. However, these studies were conducted while controlling a virtual setup [35-37] and/or while blocking the incidental (visual and auditory) feedback sources [18, 31, 36, 38]. Some recent studies showed benefits of feedback in realistic, clinical settings [32, 39-43]. However, other experiments in realistic settings failed to show functional improvements in performance $[13,19,20,44,45]$ or demonstrated some benefits of feedback only in specific conditions $[13,14,40]$.

A major confounding issue when studying the effect of artificial feedback is the existence of incidental sensory information that is already present in prosthesis control. The user can observe the prosthesis motion (visual feedback) and hear the motor sound, which together with proprioception has recently been shown to already allow for a rather good control of grasping force [46]. In addition, motor vibrations as well as mechanical interaction with the object can propagate through the socket to be felt by the user. The user can exploit these incidental information sources to close the control loop, even in the absence of an explicit somatosensory feedback. For example, in a typical myoelectric prosthesis, the velocity of closing is related to the resulting grasping force, i.e., the faster the hand closes the stronger the force produced after contact (see Fig. 2 in [47]). Therefore, the information on the closing velocity can be employed by the prosthesis user to improve the control of grasping force, as demonstrated in [48]. Importantly, the closing speed can be estimated through visual observation or by listening to the motor sound.

Although it has been demonstrated that human subjects can indeed use such information for prosthesis control, the psychometric properties of the incidental sources of feedback have not been investigated yet, in contrast to psychometric tests of the artificial feedback methods (electro- and vibrotactile stimulation) that are thoroughly documented in the literature $[16,17]$.

In the present study, we systematically investigated the quality of the incidental prosthetic feedback both objectively and subjectively. To this aim, the psychometric properties of the incidental auditory and visual feedback produced by prosthesis motion were quantified in a discrimination task (Experiment I) and then compared to 
that of a simple vibration interface in an estimation task (Experiment II). The task for the subjects was to estimate the velocity of prosthesis closing using incidental feedback. Vibratory feedback was selected for comparison as a common method to provide explicit somatosensory feedback in prosthetics. The results demonstrated that the subjects could interpret the incidental information sources rather well, and even better than the supplemental vibrotactile feedback. These observations provide insights on possible reasons for the inconsistent results related to the benefits of sensory feedback in prosthetics and thereby guide the design of novel, more effective feedback solutions.

\section{Methods}

\section{Participants}

Twelve able-bodied volunteers $(24 \pm 3$ years old, between 18 and 27 years, 6 women, 6 men) participated in the two experiments. The subjects were informed about the experiments and confirmed that they understood the experimental procedure. They gave informed consent to take part in the study and were reimbursed with 10 Euro per hour of participation. The study was approved by the ethics committee of the Universitätsmedizin Göttingen. Ten of the participants were right handed, two left handed. Four had corrected vision. All subjects had taken part in one previous study on prosthetics [46] but were otherwise naïve to prosthesis control.

\section{Experimental setup}

The current study was divided into two experiments, which were performed on different days, with Experiment I (Exp I) being at least 2 weeks before Experiment II (Exp II). The setup was similar in both experiments, as shown in Fig. 1a. It comprised 1) a prosthetic hand (Michelangelo prosthetic hand, Otto Bock Healthcare $\mathrm{GmbH}$, Vienna, AT), controlled via Bluetooth, 2) a standard desktop PC with a 22" computer screen (hidden from the subject's view) controlling the prosthesis, 3) headphones playing white noise, and 4) a wooden block. In Exp II, the setup was extended by 5) two C2 tactors with a control unit (Engineering Acoustics, US) connected to the PC via a USB port to deliver vibrations, and 6) a laptop with a mouse used by the subject to indicate answers with the right hand (as explained below). The subjects were seated in a chair with the prosthetic hand in a table-top position in front of them at a distance of approx. $0.5 \mathrm{~m}$, allowing a lateral view on the prosthesis (thumb-side, see Fig. 1). When closed using pinch grip, the prosthesis grasped a wooden block, which was fixed to the table. In Exp II, one C2 tactor was placed on the ventral aspect of the forearm of the subject's left arm. The subject placed the arm comfortably on a sideboard. The second vibrator was hanging freely in the air and was activated at the maximum intensity in each trial with vibration feedback, effectively preventing the subject to use the sound produced by the first vibrator as potential source of feedback. In addition, the subject wore headphones playing white noise.

The prosthesis and the tactors were controlled by a control-loop implemented in Matlab Simulink 2015b (Mathworks, US) using the prosthetic closed-loop test bench [49] that operated in real-time at $100 \mathrm{~Hz}$. In this study, the prosthesis was controlled by a computer, i.e. the subjects did not control the prosthesis themselves, making them passive observers. During a single trial, the prosthesis closed with constant velocity, simulating a routine-grasping protocol [46]. Across trials, the closing velocity was changed according to the psychometric test procedure (explained below). In Exp I, the subjects verbally reported their discrete answers to the experimenter, whereas in Exp II they used the mouse to indicate the answer via a Matlab GUI.

\section{Experiment I: discrimination task}

The aim of the first experiment was to assess the just noticeable difference (JND) in the incidental feedback using a staircase procedure. The JND is the minimal change in the amplitude of the feedback variable that can be perceived by the subject [50], therefore determining the effective resolution of the feedback channel. In the context of the present study, the JND corresponds to the minimal change in the prosthesis closing velocity that can be perceived by looking at the prosthesis (visual JND) and/or by listening to the prosthesis sound (auditory JND).

The experiment started with a familiarization phase in which the subjects watched as the prosthesis closed 10 times. The closing velocities changed from 20 to $100 \%$ of the maximum velocity (in steps of $20 \%$ ), which corresponds to absolute average closing speeds between 38 $\mathrm{mm} / \mathrm{s}$ and $324 \mathrm{~mm} / \mathrm{s}$ (see Fig. 8 in the Appendix). This sequence was repeated twice. The subjects could see and hear the prosthesis while it closed.

The JND was assessed using a transformed 1 up / 2 down staircase procedure in a two-alternative forcedchoice (= 2AFC) task, which targets $70.71 \%$ correct performance [50]. Each step of the staircase procedure comprised two trials of prosthesis closing. The prosthesis closed once at a fixed predefined velocity (standard value) and once at a velocity (comparison value) that was changed adaptively across trials based on the participant's previous answers. In each trial, the standard and comparison value were provided in random order. The prosthesis stayed for $1 \mathrm{~s}$ in the opened or closed position, before closing or opening again. The comparison value was always higher than the standard value. The subjects observed the prosthesis using the provided 


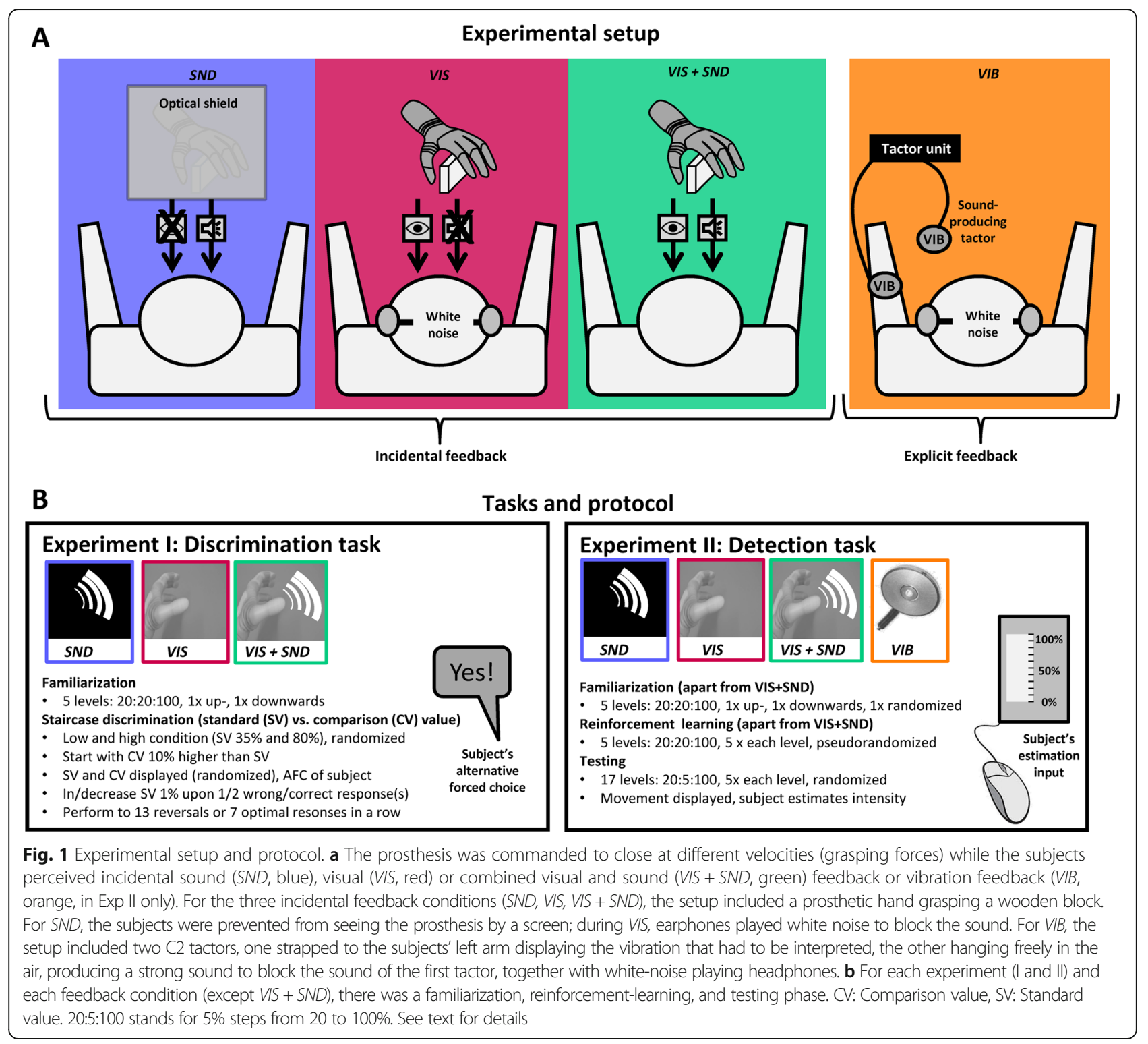

auditory information (SND), visual information (VIS), and visual and auditory information $(V I S+S N D)$, depending on the condition, and they were then asked to report in which trial the prosthesis closed faster. If subjects answered correctly two times in a row, the comparison value was reduced by $1 \%$. If they made a mistake, it was increased by $1 \%$. The first five steps of the staircase were used for reinforced learning, where the experimenter revealed the correct response, to make sure that the subjects understood the task. The initial comparison velocity was set to be $15 \%$ higher than the standard one, which was a large difference and hence simple to discriminate. After the reinforcement learning, the normal staircase procedure resumed, i.e., the subjects received no more feedback on the correct answer. The staircase procedure was terminated if 13 reversals occurred, with a reversal being the change of the comparison value from increasing to decreasing or vice versa (see Fig. 2, VIS for an example). The JND (staircase outcome) was then calculated as the difference between the mean of the last 10 reversals and the standard value. The procedure was also terminated if the comparison value was only $1 \%$ higher (i.e., as low as possible) than the standard value 7 times in a row (see Fig. 2, SND and $V I S+S N D$ for an example). In this case, the JND was set to $1 \%$. To assess the JND at both low and high velocities, the staircase was performed two times, at the standard values of 35 and $80 \%$, respectively (order pseudorandomized across subjects), that correspond to absolute average closing speeds of $72 \mathrm{~mm} / \mathrm{s}$ and $231 \mathrm{~mm} / \mathrm{s}$ (see Fig. 8 in the Appendix). 

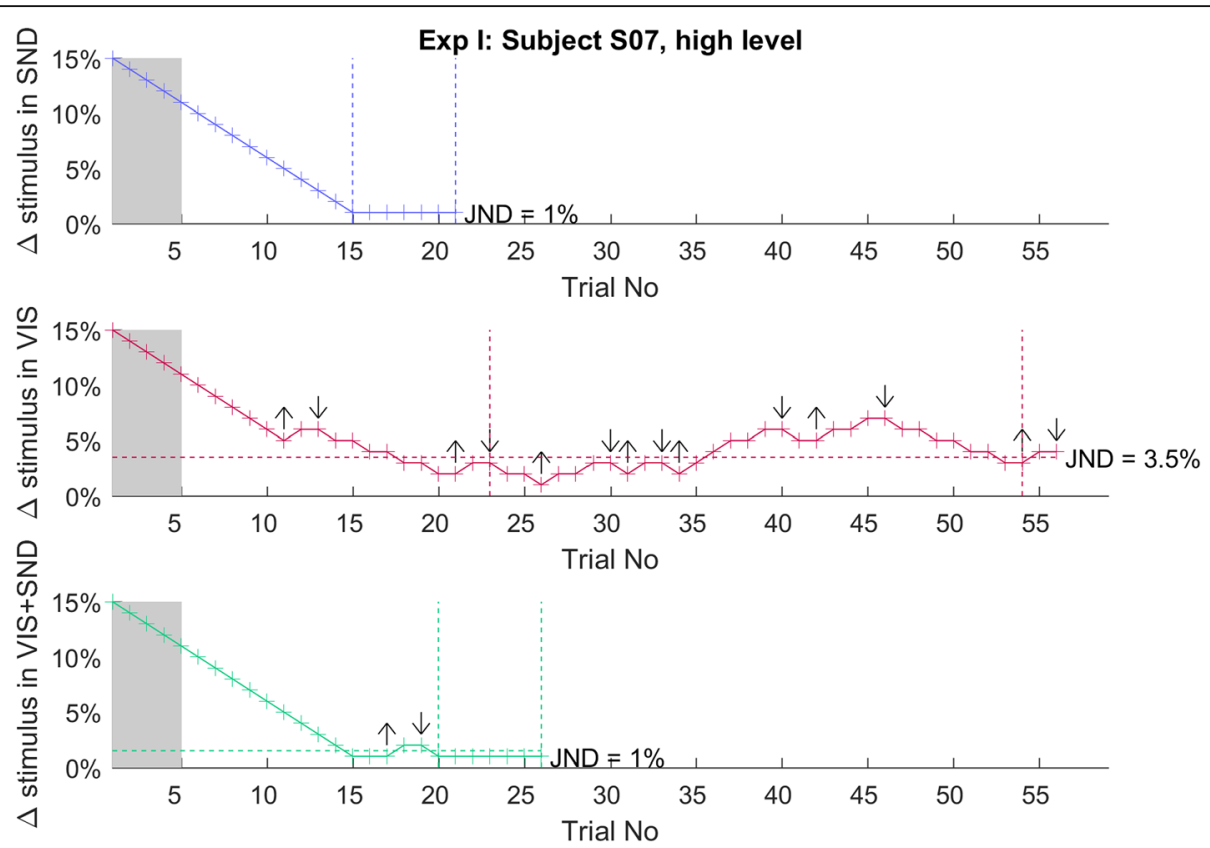

Fig. 2 A staircase procedure for one representative subject in Exp I. The time course of the difference $(\Delta)$ between comparison and standard value recorded in Exp I across trials of a staircase procedure for Subject 7 at the high standard-value level (80\%) for the three feedback conditions SND (top, blue), VIS (middle, red), and VIS + SND (bottom, green). The first 5 trials (grey underlay) reflect the reinforcement-learning phase. After that, wrong answers led to a $1 \%$ increase of the comparison stimulus, while two correct answers were needed for a $1 \%$ decrease (1 up/2 down staircase). The 10 reversals (indicated by an up-downward arrow) in the area between the vertical dashed lines (4th to 13th reversal) were used to calculate the JND, which is indicated by a horizontal dashed line. If the subject's response was correct 7 times in a row at a 1\% difference between comparison and standard stimulus, the JND was set to $1 \%$ and the run was terminated (see SND and VIS + SND)

The JND was measured in several conditions with different implicit feedback. In $S N D$ the vision was blocked by a screen in front of the prosthesis, which made only the closing sound available. In VIS, the subjects could see but not hear the prosthesis since the sound was blocked by noise-cancelling earphones playing white noise. In the final condition $(V I S+S N D)$, the subjects could normally hear and see the prosthesis. The order of the feedback conditions was pseudorandomized across subjects. Generally, the subjects were encouraged to focus on every detail of the closing and grasping process that they deemed could be useful to evaluate the presented closing velocity. This included the interaction between the wooden block and the prosthesis, the duration of the closing process, the frequency and intensity of the sound, etc. All these possibilities were explained to the subject beforehand.

The JND was computed for each subject, feedback condition, and standard-value velocity. As KolmogorovSmirnov tests showed that the data were not normally distributed, non-parametric tests were used and medians and interquartile ranges are reported in the manuscript. To compare the three feedback conditions, the subjects' JNDs were averaged across the two standard stimuli and a Friedman test was applied. Upon significance, the conditions were compared pairwise using three Bonferroni- corrected Wilcoxon signed-rank tests. For comparison between low and high velocities separately for each feedback condition, three Bonferroni-corrected Wilcoxon signed-rank tests were performed. The threshold for statistical significance was set to $p<0.05$.

\section{Experiment II: estimation task}

The aim of the second experiment was to assess how well the subjects could estimate the prosthesis closing velocity using different sources of incidental feedback. In this case, the prosthesis closed at a constant but arbitrary velocity and the subjects were asked to provide an estimate of the closing speed (absolute estimation versus relative discrimination in Exp I). In addition, the subjects' ability of estimating the intensity/frequency of vibrotactile stimulation was assessed, a method which is commonly used in prosthetics to implement sensory feedback [10].

The experiment comprised four feedback conditions, three with implicit (SND, VIS, and VIS + SND), and one with vibrotactile feedback $(V I B)$. Each condition comprised a familiarization phase, a reinforcement-learning phase, and a testing phase. The only exception was VIS + $S N D$, where only the test phase was implemented to assess the potential fusion of information from the incidental feedback sources. 
During the familiarization phase in SND and VIS, the prosthetic hand was closed at five velocities $(20,40,60$, 80 , and $100 \%$ ) first in increasing, then in decreasing, and finally in random order. In the reinforcement-learning phase, the prosthesis closed randomly five times at each of these velocities (25 trials). After each trial, the subjects were asked to estimate the closing speed and the experimenter then informed them about the correct response. In the testing phase (correct answer not provided), the prosthesis closed at 17 velocities $(20-100 \%$ with $5 \%$ steps), five times per velocity in random order. Importantly, the subjects were not informed about the discrete step-sizes (5\%) but were told that any velocity between 20 and $100 \%$ might occur, in order to eliminate a bias towards the $5 \%$ steps. After the prosthetic hand closed, the subjects reported their closing-speed estimate by a mouse click to an analog scale (20-100\%) shown at the laptop.

For the vibrotactile condition, the protocol was similar except that the task was to estimate the intensity/frequency of a vibrotactile stimulus (the prosthesis was not used). In each trial, a $2.5 \mathrm{~s}$ vibration was delivered using a C2 tactor fixed on the subjects' left arm. The same levels and number of trials were used as in VIS and $S N D$. The levels were implemented by simultaneously changing the frequency and intensity of stimulation. The dual-parameter modulation was used to facilitate the discrimination. The frequency (1-100\%) ranged from 30 $\mathrm{Hz}$ to $270 \mathrm{~Hz}$, and the intensity $(1-100 \%)$ was chosen between 8 and $71 \%$ of the maximum intensity. Therefore, this condition was equivalent to using vibration feedback to transmit the prosthesis closing velocity via linear mapping.

The order of the four feedback conditions was randomized between subjects. However, the VIS + SND was always placed after VIS and SND such that the subjects received the same amount of learning in each of the two modalities before the fusion of modalities $(V I S+S N D)$ was tested. To investigate to which extent specific features were important when interpreting the feedback in each condition, the subjects were asked to indicate how much they relied on each feature using a visual-analog scale $(0-10)$ in a questionnaire. They indicated how much they had focused on the speed of closing, duration of closing, and mechanical interaction between the prosthesis and the object for VIS, duration, frequency, and loudness of sound for $S N D$, and frequency and intensity of vibration for VIB. In addition, in each condition they were asked to estimate their average absolute deviation from the correct value, to assess how confident they were in their estimation.

For evaluation, the success rate for the reinforcementlearning phase and the estimation errors in the testing phase were calculated for each trial, separately for each subject, level, and feedback condition. Again, nonparametric statistics were used. For the analysis of the reinforcement learning, the subjects' success rate, averaged across the five tested levels, was compared across the three trained feedback conditions using a Friedman test and, upon significance, three Bonferroni-corrected Wilcoxon signed-rank tests were applied. In the testing phase, the median of the estimated value was computed for each level and feedback condition, reflecting whether and how much the subject over- or underestimated the correct level. For each level (except for the lowest and highest where over- and undershooting were not possible, respectively) and feedback condition, Bonferroni corrected Wilcoxon signed-rank tests were used to assess whether the subjects consistently over- or underestimated the level, by subtracting the estimated from the correct value and testing whether that value differed significantly from zero. Then, by pooling the absolute errors of all respective trials, the median absolute error (MAE) was determined for each subject and feedback condition, independently of the level. To test for differences between feedback conditions, a Friedman test and, upon significance, six Bonferroni-corrected Wilcoxon signed-rank tests on the MAE were used.

For analysis of the subjective results from the questionnaire, for both $S N D$ and VIS as well as separately for the acoustic and the visual features in VIS + SND, a Friedman test was applied and, upon significance, three Bonferroni-corrected Wilcoxon signed-rank tests were used for pairwise comparison. For VIB, a single Wilcoxon signed-rank test was used (only two features available). Six Bonferroni-corrected Wilcoxon signed-rank tests compared whether the feature usage differed when using one-feedback modality (SND or VIS) instead of full feedback $(V I S+S N D)$, e.g., the subjective reliance on the auditory duration of the prosthesis closing was compared between $S N D$ and $V I S+S N D$ condition. In addition, a dominance towards visual versus auditory features was explored by comparing the amount of reliance on the visual versus auditory features in $V I S+S N D$, indicating which modality captured more attention when the full implicit feedback was available. To that end, in the VIS + SND condition for each subject the most strongly used auditory and visual features were selected across the three possible features per modality and these two were compared via a Wilcoxon signed-rank test.

\section{Results}

\section{Experiment l: discrimination task}

A representative example of a staircase trial is shown in Fig. 2. In general, at the high level of the standard stimulus, the subjects were able to discriminate the smallest possible change in prosthesis closing velocity (1\%) in $S N D$ and VIS + SND conditions. The staircase sequence 
"saturated" at the difference of $1 \%$ in 9 and 10 out of the 12 subjects, respectively. However, this did not happen for any of the subjects in the VIS condition, and it was also uncommon at the low level of the standard stimulus regardless of the feedback condition (i.e., only a single subject per condition succeeded in saturating the staircase). Nevertheless, all feedback conditions resulted in a small JND with a median value of less than $4 \%$. The summary results are shown in Fig. 3. The visual feedback was characterized with slightly higher JNDs. With median JND of 3.7 and 3.5\% for the low and high level, respectively, VIS led to low but still significantly higher JNDs compared to $S N D$ (1.5 and 1\%, respectively; $p<$ $0.001)$ and $V I S+S N D$ (1.5 and $1 \%$, respectively; $p=$ $0.007)$. The median JND in the latter two conditions was very close or even equal to the smallest possible change in velocity (1\%). The JND was slightly but significantly smaller at the high compared to the low standardstimulus level for $S N D(p=0.006)$ and VIS + SND $(p=$ 0.004 ), while no significant difference was observed for VIS. Generally, these data show a surprisingly good discrimination no matter which type of implicit feedback was provided to the subjects.

\section{Experiment II: estimation task}

During reinforcement learning, the success rates (median [interquartile range]) were $92 \%$ [8\%] in $S N D, 88 \%$ [82\%] in VIS, and 74\% [18\%] in VIB. Hence, the performance in VIB was worse compared to the two other conditions.

The data from the testing phase of one representative subject are shown in Fig. 4, where the estimated versus correct closing velocities (SND, VIS, VIS +SND) and vibration levels $(V I B)$ are plotted against each other for all feedback conditions and tested levels. The points are scattered around the optimal-performance line (black line), with a slightly worse quality of estimation in VIB.

The overall results are shown in Figs. 5 and 6. The medians of the estimated values followed consistenly the optimal-performance line (in black) across levels (Fig. 5). Statistically significant deviations from the line were not observed at any level for $S N D$ and VIS, at only two levels for $V I S+S N D$, and at 5 out of 15 levels for $V I B$, indicating a general trend to underestimate the levels between 40 and $70 \%$ in this condition. Figure 6 shows the boxplots of the median absolute errors (MAE) across subjects (obtained by pooling trials across tested levels) for all feedback conditions. The performance was similar in SND (5.2\% MAE) and VIS + SND (5.1\% MAE). The estimation error in VIS + SND was significantly smaller than in VIS $(6.7 \% ; p=0.005)$ and VIB $(8.4 \% ; p<0.001)$ and the error in $S N D$ was significantly smaller than in $V I B$ $(p=0.002)$. No significant difference in the quality of estimation was found between VIS and VIB.

Figure 7 summarizes the results of the questionnaire in which the subjects reported on the relevance assigned to different features of the feedback sources. Regarding the auditory features, most subjects seemed to focus on closing duration (median $80 \%$ for $S N D$ and $70 \%$ for $V I S+S N D$ ) and frequency (80\% for $S N D$ and $80 \%$ for $V I S+S N D)$ instead of intensity ( $45 \%$ for $S N D$ and $35 \%$ for VIS + SND). However, this trend was not significant neither in SND nor in VIS + $S N D$ likely due to high variability across subjects. Regarding visual features in VIS (Friedman test $p<0.001$ ) and VIS + $S N D$ (Friedman test $p<0.001$ ), the subjects were significantly more attentive to the duration $(90 \%$ for VIS, $p<0.001$, and

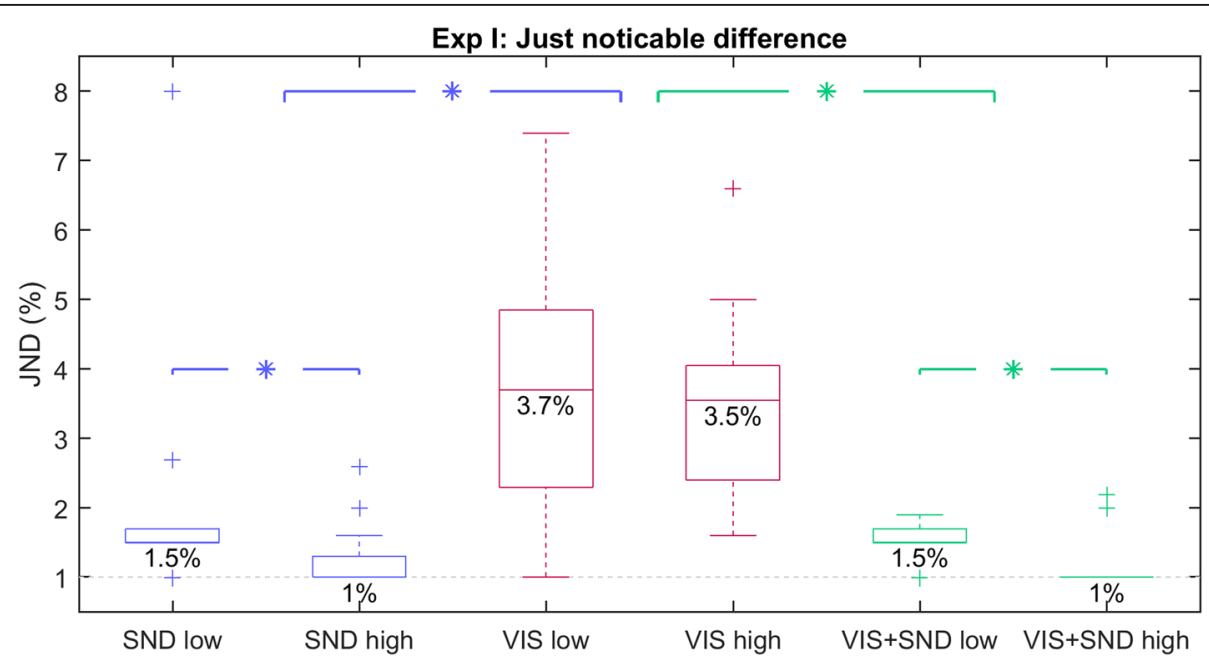

Fig. 3 Overall JNDs for Exp I. The boxplots represent the overall JNDs across subjects for each feedback condition (SND in blue, VIS in red, VIS + SND in green) at the low (left) and high (right) standard-stimulus level, with the horizontal line indicating the median JND (also given as number), the box the interquartile range, the whiskers the range, and the pluses the outliers. Statistically significant differences between feedback conditions or between low and high levels within one feedback condition are depicted by asterisks 


\section{Exp II: Estimated closing velocities per level, Subject 08}
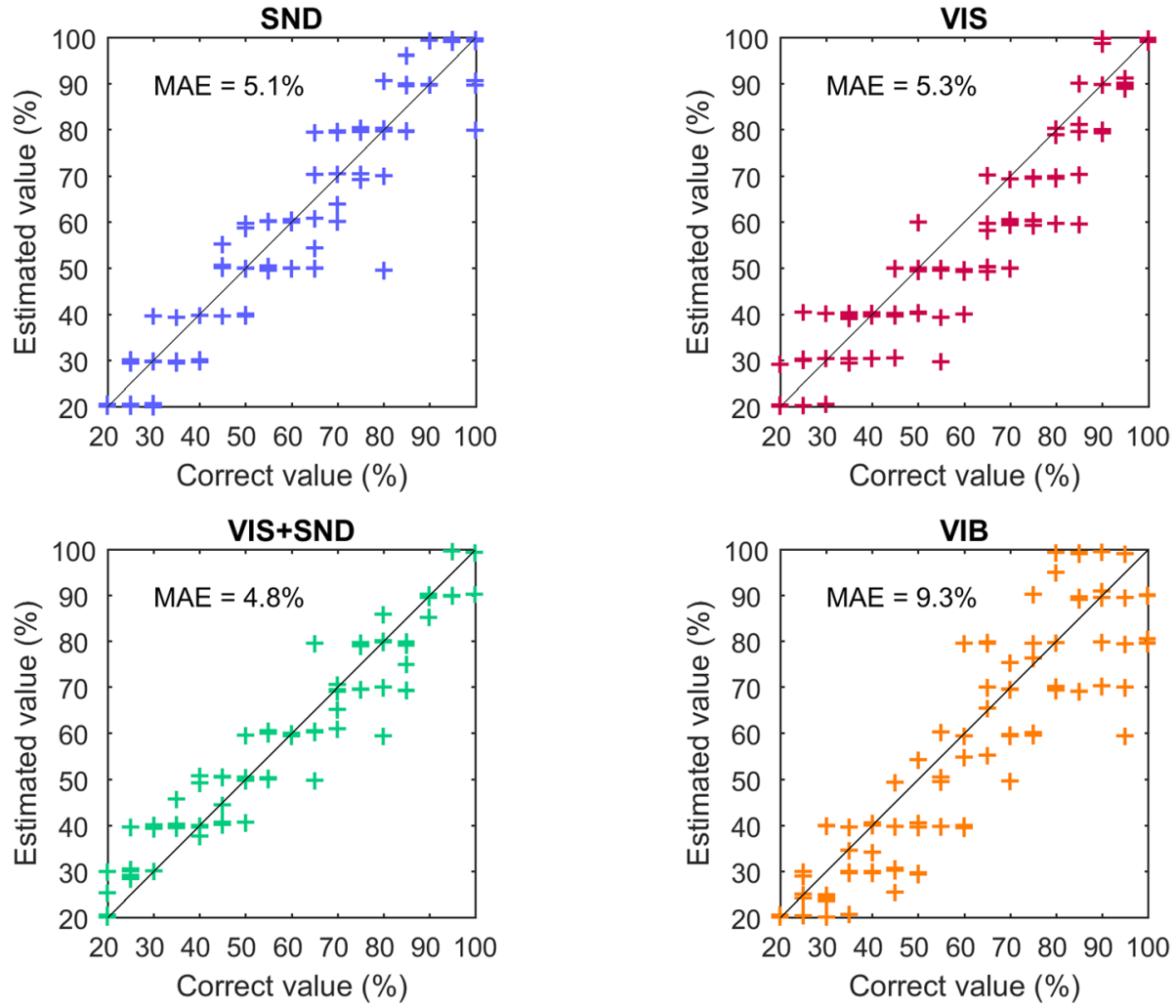

Fig. 4 The results from the testing phase of one representative subject in Exp II. The estimated closing velocity (VIS, SND, VIS + SND) and vibration intensity (VIB) plotted against the correct stimulus value for Subject 8. The black line is the reference that would indicate the perfect performance (all responses correct)

$70 \%$ for $V I S+S N D, p=0.001)$ and closing speed $(60 \%$ for VIS, $p=0.001$, and $70 \%$ for VIS $+S N D, p<0.001)$ than to the interaction between prosthesis and wooden block (10\% for $V I S$ and $0 \%$ for $V I S+S N D$ ), which was hardly exploited at all. No significant differences in used features between full $(V I S+S N D)$ and partial $(S N D$ or VIS) feedback were observed, except for a higher focus on the closing duration in $V I S$ compared to VIS + SND (shown by a black asterisk in Fig. 7; $p=0.002)$. In $V I S+S N D$, the subjects concentrated similarly on visual and auditory features, with none of the two modalities being rated significantly higher than the other. For the vibratory task, the subjects clearly focused significantly more on the intensity (median 100\%) than on the frequency information $(10 \% ; p<0.001)$. Concerning the subjective estimation of performance, they reported a subjective MAE of $10 \%$ (IQR 6\%) for SND, 17\% (IQR 8\%) for VIS, 9\% (IQR 7\%) for $V I S+S N D$, and 15\% (IQR 12\%) for VIB.

\section{Discussion}

The present study investigated the psychometric properties of the incidental feedback in prosthetic grasping. The quality of feedback sources inherent to a prosthetic device (vision and sound) was assessed by conducting psychometric tests and it was compared to the quality of feedback provided by a vibration motor, which is a common solution to transmit somatosensory feedback in prosthetics. The experimental results demonstrated that the visual and auditory feedback of the prosthesis motion could be used to estimate the closing speed with high reliability. In addition, the estimation using sound and vision alone or in combination was significantly better compared to the vibration feedback.

With sound and vision combined or only sound alone, the obtained JND was below $2 \%$ at both high and low closing speeds. With vision alone, the JND was somewhat higher (below 4\%), which is in agreement with who reported that auditory feedback is more useful than visual feedback for temporal discrimination. The present study therefore demonstrates that the inherent resolution of the incidental feedback sources is truly remarkable. Only by relying on the incidental feedback, the subjects were capable of discriminating changes in the prosthesis closing velocity that are close to the minimum increment/decrement in velocity that can be commanded to the prosthesis (1\%). In addition to detecting a change in velocity, the subjects could estimate the 


\section{Exp II: Median estimated value per level}
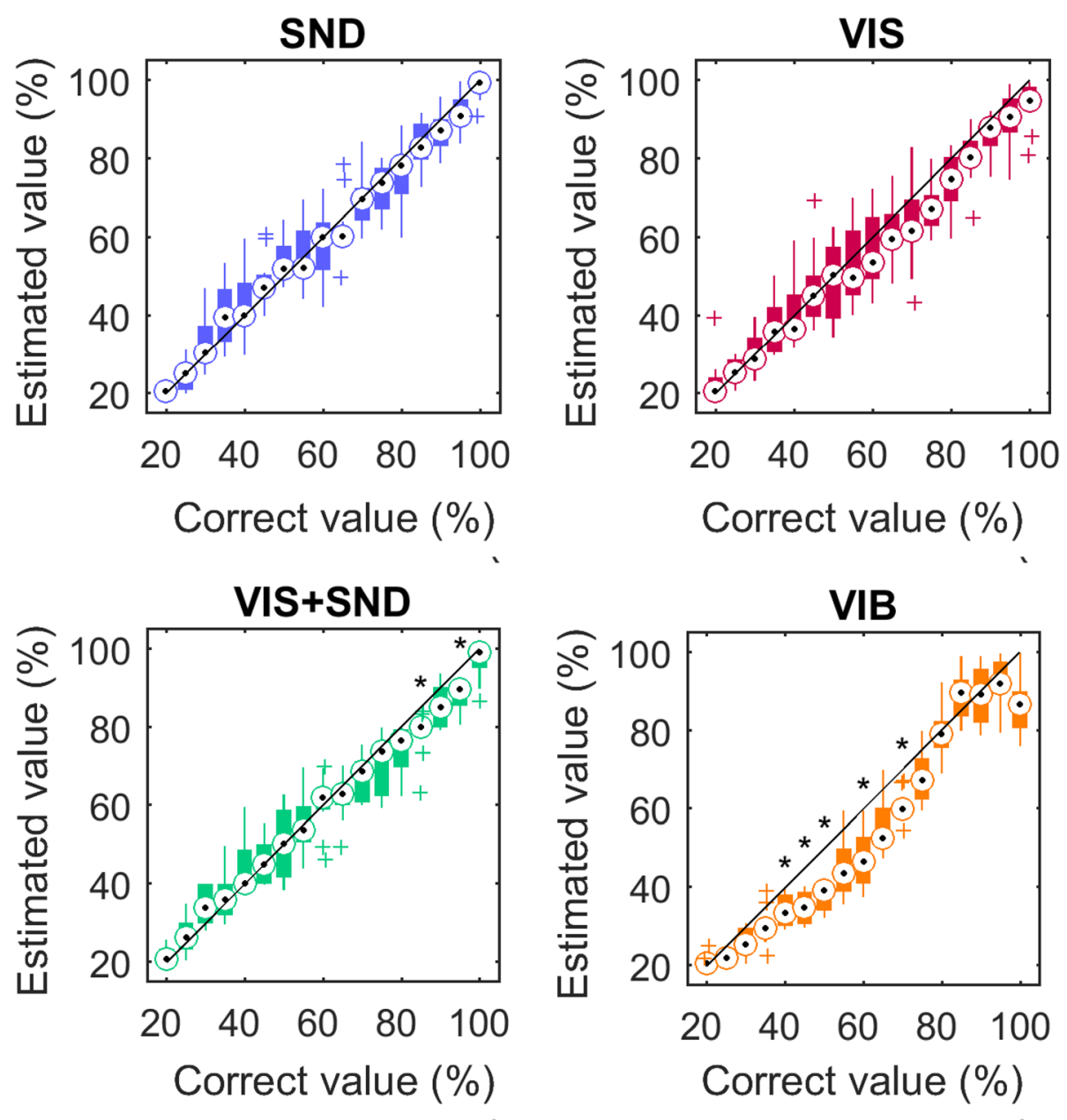

Fig. 5 Median estimation per level for Exp II. Estimated value, i.e. estimated closing velocity/vibration, plotted against correct stimulus, i.e. generated velocity/vibration, with every boxplot representing the median (circle), interquartile range (box), range (line), and outliers (pluses) of all subjects' medians for one level. Each subpanel represents one of the feedback conditions (see title). The black line is the reference line, which would reflect perfect responses. Significant differences $(p<0.05)$ from that line are indicated by asterisks

absolute value of the closing speed with high precision. When estimating a closing speed randomly selected from the full working range, the average error was around 5\% for SND and VIS + SND and around 7\% for VIS. As explained in Introduction, the estimation of prosthesis closing velocity can be used to control grasping force [48]. The present study shows that the incidental feedback can provide such an estimate rather precisely, and this explains why the incidental sources can be so useful for prosthesis control [46].

The combined visual and auditory feedback resulted in the best performance, as indicated by both lowest MAE and low variability across subjects. This demonstrates that the subjects could fuse the incidental sources of feedback to improve the estimation of the prosthesis state. The studies of human motor control have shown that human subjects can integrate sensory information from multiple sources based on their relative uncertainty [51-53].

The present study demonstrated that the inherent prosthesis feedback could provide reliable closing-speed information to the prosthesis user after only a brief training $(<5 \mathrm{~min})$. When the same information was transmitted using only vibration feedback, the quality of estimation was significantly worse (MAE 8\%). Importantly, the results for VIB condition might be different if another coding scheme would be used. We have used a linear mapping since this is a simple and therefore a common choice in the literature $[36,54,55]$. In reality, the mapping from the intensity of stimulation to the 


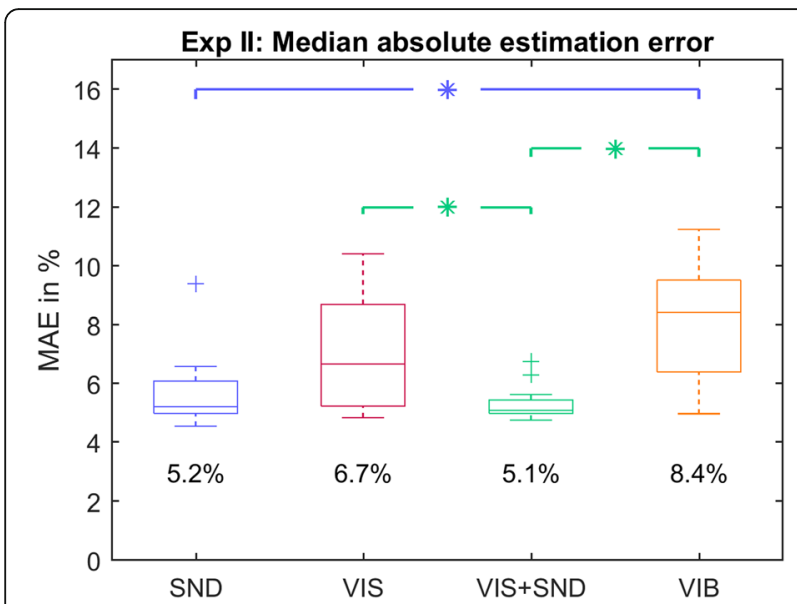

Fig. 6 Overall median absolute estimation (MAE) error for Exp II. The MAE between estimated stimulus and correct stimulus with every boxplot representing the median (horizontal line), interquartile range (box), range (dashed line), and outliers (pluses) of all subjects' MAE (obtained after pooling the absolute errors over all respective trials). Significant differences $(p<0.05)$ between feedback conditions are indicated by asterisks

perceived intensity follows a power function $[17,56]$. And indeed, such a trend can be seen in Fig. $5(V I B)$, where the subjects underestimated consistently in the middle range of intensities. This effect, although significant, was not substantial, possibly because we have modulated the frequency in addition to the intensity to further facilitate the discrimination. Nevertheless, better estimation (e.g., less undershooting) in the VIB condition might be obtained if a different transfer function would be used; this remains to be tested.

The above results might indicate that adding vibration as a supplemental feedback to communicate the variables that can be already estimated from the intrinsic sources directly (closing velocity) or indirectly (grasping force) could have limited impact on performance of prosthesis control. The intrinsic feedback seems to be sufficient so that there is not much margin for improvement. Of course, this assumes that the subject can indeed observe the prosthesis while closing. Also, the performance of $V I B$ was worse already in the learning phase, which was rather short in the present study. It would be interesting to investigate the trends as well as the final performance in each feedback modality after a prolonged learning.

There was a substantial variability in the subjective reports about the relevance of different features of the feedback sources. Nevertheless, in all the incidental feedback conditions, the subjects focused on the temporal aspects of the feedback stimulus, namely, the stimulus duration. On the contrary, the cues from the mechanical interaction between the prosthesis and the object seem not to be exploited. Finally, although the sound frequency was appreciated by the subjects in the acoustic feedback condition, when using vibration feedback, they focused substantially more on the intensity. This could be due to the frequency range of the $\mathrm{C} 2$ tactor, which does not include low frequencies $(<30 \mathrm{~Hz})$, whereas the intensity was modulated between 8 and $71 \%$. Interestingly, when

\section{Exp II: Features subjectively used for task completion}

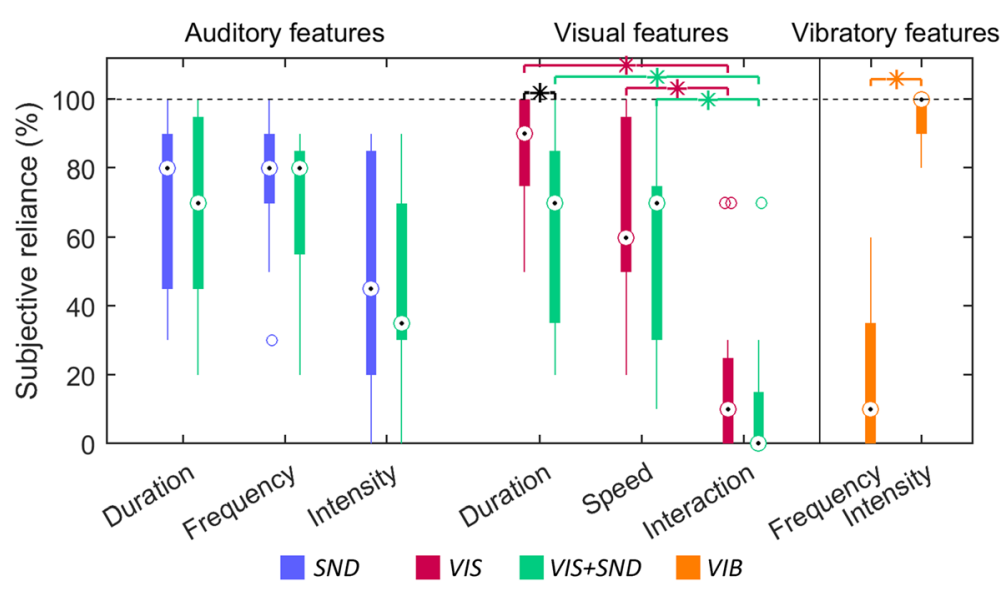

Fig. 7 Subjective feature evaluation for Exp II. Boxplots reflecting the subjectively estimated reliance on the different available feedback sources for task completion in the four feedback conditions (colour-coded with blue for SND, red for VIS, green for VIS + SND, and orange for VIB). Possible feedback sources for closing-velocity estimation were the duration of a stimulus (visual and/or auditory), the speed (visual), frequency (auditory), and intensity (auditory) of the closing hand, and the interaction between the fingers of the prosthesis and the wooden block (visual), while for vibration estimation the frequency and intensity of the tactor could be used. Every boxplot represents the median (circle), interquartile range (box), range (line), and outliers (pluses) of all subjects' responses. Significant differences within feedback conditions are shown by asterisks in the respective color, black asterisks show differences between single-modality and full feedback tasks 
estimating their own performance, the subjects ranked the conditions according to the objectively measured results (i.e., SND and VIS + SND performed better than VIS and $V I B)$. However, they reported a higher subjective uncertainty, i.e., the perceived estimation error was greater than experimentally observed.

The present study is an important initial step towards understanding the interaction between incidental and explicit feedback in closed-loop prosthetics. In another prosthesis, the vision and sound feedback would be different in quality and/or quantity (e.g., sound pitch and loudness), but the main principle would still hold (e.g., the movement speed and therefore sound associated to force).

\section{Limitations}

In real life scenarios, the usefulness of incidental (visual and auditory) feedback can decrease in the presence of overlaying and distracting sensory inputs, e.g. in noisy environments or when the visual attention is diverted from the prosthesis. Also, wearing a cosmetic glove over the prosthesis, which is usual in clinical applications, might decrease the loudness and discriminability of the auditory information. In this case, supplemental vibroor electrotactile feedback might be beneficial since it is transmitted through a different sensory channel (sense of touch).

The experimental task in the present study resembled routine grasping, in which the prosthesis closes at a constant velocity to produce proportional force upon contact. However, modulating the force after the prosthesis has closed around an object is a different task, in which supplemental force feedback, delivered using vibration or other methods, is likely to be useful. In this case, different sources of feedback might be relevant. For example, if the prosthesis holds a compliant object while closing, the subjects could estimate the grasping force from the amount of object deformation.

In the present experiment, the computer sent a command to the prosthesis to close at a constant velocity. In real-life application, the prosthesis will be controlled by a user generating myoelectric commands. The myoelectric signals are known to be variable and this is likely to cause variability in the prosthesis closing speed (even if the user would like to produce a constant velocity). This can challenge the estimation of prosthesis variables from the incidental sources. Nevertheless, recent studies show that incidental feedback can be useful despite this uncertainty $[46,48]$. However, it would be useful to measure the psychometric parameters in this context, namely, during realistic proportional control and feedback. In this case, a proprioceptive information from the sense of muscle contraction could be used additionally to vision and audition [46]. Finally, the results reported here were observed in naïve able-bodied subjects; the implications obtained, however, should be even more relevant for experienced prosthesis users, who had enough time and practice to learn to interpret the prosthesis sound and movement.

\section{Conclusion}

The present study shows that the implicit feedback coming from a hand prosthesis can be an important source of information when estimating the prosthesis state. The subjects were able to accurately discriminate (experiment I) and estimate (experiment II) the velocity of prosthesis closing using vision and sound, and they could even fuse the two incidental feedback sources to improve the estimation quality. This is a strong indication that the feedback, which is intrinsically available from the prosthesis, can be usefully exploited for the control of prosthesis grasping. This point should be considered when designing supplemental feedback provided through sensory substitution (e.g., electro and vibrotactile stimulation). If supplemental feedback is to be efficient, it needs to be of a higher quality or should transmit information that is not already available implicitly (e.g., EMG feedback $[41,57,58]$ ).

\section{Appendix}

To measure the prosthesis closing speed, the hand was mounted on a vice and fully opened in palmar grasp. A Matlab program was implemented that repeatedly closed and opened the prosthesis through a full range of speeds. The normalized prosthesis commands in the range between 20 and $100 \%$, in steps of 5\%, were repeated three times and the time needed for closure from the fully open prosthesis (12 $\mathrm{cm}$ aperture according to the manufacturer) to the fully closed prosthesis was measured. Then, the average closing speed was computed by dividing the

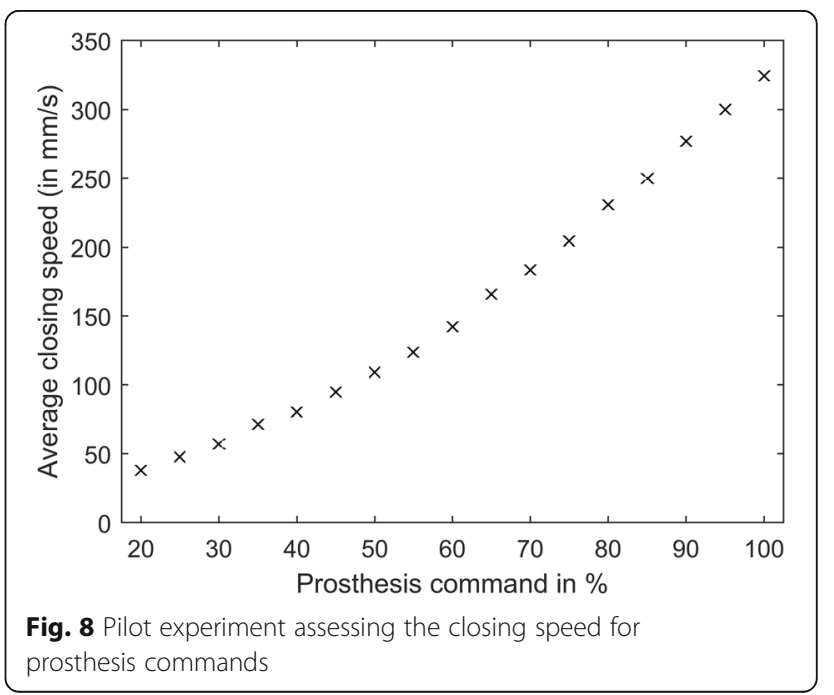


aperture $(12 \mathrm{~cm})$ by the mean closing time per prosthesis command. The profile of obtained closing speeds is depicted in Fig. 8. It can be seen that the two standard speeds used for JND measurements correspond to the two segments of the profile with different slopes.

\section{Abbreviations}

CV: Comparison value; Exp I: Experiment I; Exp II: Experiment II; JND: Just noticeable difference; MAE: Median absolute error; SND: Acoustic feedback; SV: Standard value; VIB: Vibrotactile feedback; VIS + SND: Combined feedback; VIS: Visual feedback

\section{Acknowledgements}

None.

\section{Authors' contributions}

MS, CN, and SD designed the experiment. MS and BM performed the experiment. MS, SD, and BM analysed and interpreted the data. MS, CN, and SD prepared the manuscript. All authors provided critical feedback on the manuscript, read, and approved the final manuscript.

\section{Funding}

This work was supported by a grant from the Bundesministerium für Bildung und Forschung (BMBF) (INOPRO-16SV7656) and Independent Research Fund Denmark (ROBIN-8022-00243A).

\section{Availability of data and materials}

The datasets used and/or analysed during the current study are available from the corresponding author on reasonable request.

\section{Ethics approval and consent to participate}

The study was approved by the ethics committee of the Universitätsmedizin Göttingen. The subjects provided written informed consent before participation.

\section{Consent for publication}

Subjects provided written informed consent for publication before participation.

\section{Competing interests}

The authors declare that they have no competing interests.

\section{Author details}

'Department of Biotechnology, University for Applied Sciences Hamburg, Hamburg, Germany. ${ }^{2}$ Advanced Rehabilitation Technology (ART) Lab, Department for Trauma Surgery, Orthopaedics and Plastic Surgery, Universitätsmedizin Göttingen (UMG), Göttingen, Germany. ${ }^{3}$ Department of Computer Science, Eberhard-Karls University Tübingen, Tübingen, Germany. ${ }^{4}$ Department of Bioengineering, Imperial College London, London, UK. ${ }^{5}$ Department of Health Science and Technology, Center for Sensory-Motor Interaction, Aalborg University, Aalborg, Denmark.

Received: 11 June 2019 Accepted: 13 November 2019

Published online: 10 December 2019

\section{References}

1. Johansson RS. How Is Grasping Modified by Somatosensory Input? In: Motor Control: Concepts and Issues; 1991. p. 331-55.

2. Nowak DA, Glasauer S, Hermsdörfer J. How predictive is grip force control in the complete absence of somatosensory feedback? Brain. 2004;127(1): 182-92.

3. Rothwell JC, Traub MM, Day BL, Obeso JA, Thomas PK, Marsden CD. Manual motor performance in a deafferented man. Brain. 1982;105(3):515-42.

4. Parker $P$, Englehart $K$, Hudgins B. Myoelectric signal processing for control of powered limb prostheses. J Electromyogr Kinesiol. 2006;16(6):541-8.

5. Vujaklija I, Farina D, Aszmann OC. New developments in prosthetic arm systems. Orthop Res Rev. 2016;8(July):31-9.

6. Scott RN, Parker PA. Myoelectric prostheses: state of the art. J Med Eng Technol. 1988;12(4):143-51.
7. Peerdeman B, Boere D, Witteveen $H$, Hermens $H$, Stramigioli S, et al. Myoelectric forearm prostheses: state of the art from a user-centered perspective. J Rehabil Res Dev. 2011;48(6):719-37.

8. Childress DS. Closed-loop control in prosthetic systems: historical perspective. Ann Biomed Eng. 1980;8(4-6):293-303.

9. Hebert JS, Olson JL, Morhart MJ, Dawson MR, Marasco PD, Kuiken TA, et al. Novel targeted sensory Reinnervation technique to restore functional hand sensation after Transhumeral amputation. IEEE Trans Neural Syst Rehabil Eng. 2014;22(4):765-73.

10. Schofield JS, Evans KR, Carey JP, Hebert JS. Applications of sensory feedback in motorized upper extremity prosthesis: a review. Expert Rev Med Dev. 2014;13(5):1-13.

11. Svensson P, Wijk U, Björkman A, Antfolk C. A review of invasive and noninvasive sensory feedback in upper limb prostheses. Expert Rev Med Dev. 2017;14(6):439-47.

12. Stephens-Fripp B, Alici G, Mutlu R. A review of non-invasive sensory feedback methods for transradial prosthetic hands. IEEE Access. 2018;6: 6878-99.

13. Chatterjee A, Chaubey P, Martin J, Thakor N. Testing a prosthetic haptic feedback simulator with an interactive force matching task. JPO J Prosthetics Orthot. 2008:20(2):27-34.

14. Brown JD, Paek A, Syed M, O'Malley MK, Shewokis PA, Contreras-Vidal JL, et al. An exploration of grip force regulation with a low-impedance myoelectric prosthesis featuring referred haptic feedback. J Neuroeng Rehabil. 2015;12:104

15. Bach-y-Rita P, Kercel SW. Sensory substitution and the human-machine interface. Trends Cogn Sci. 2003;7:541-6.

16. Szeto AYJ, Saunders FA. Electrocutaneous Stimulation for Sensory Communication in Rehabilitation Engineering. IEEE Trans Biomed Eng. 1982;4:300-8.

17. Kaczmarek KA, Webster JG, Bach-y-Rita P, Tompkins WJ. Electrotactile and vibrotactile displays for sensory substitution systems. Biomed Eng IEEE Trans. 1991;38(1):1-16.

18. Witteveen HJB, Droog EA, Rietman JS, Veltink PH. Vibro- and electrotactile user feedback on hand opening for myoelectric forearm prostheses. IEEE Trans Biomed Eng. 2012;59(8):2219-26.

19. Saunders I, Vijayakumar S. The role of feed-forward and feedback processes for closed-loop prosthesis control. J Neuroeng Rehabil. 2011;8(1):60.

20. Cipriani C, Zaccone F, Micera S, Carrozza MC. On the shared control of an EMG-controlled prosthetic hand: analysis of user-prosthesis interaction. IEEE Trans Robot. 2008;24(1):170-84

21. Antfolk C, D'Alonzo M, Rosén B, Lundborg G, Sebelius F, Cipriani C. Sensory feedback in upper limb prosthetics. Expert Rev Med Dev. 2013; 10(1):45-54.

22. Battaglia E, Clark JP, Bianchi M, Catalano MG, Bicchi A, O'Malley MK. The Rice Haptic Rocker: skin stretch haptic feedback with the Pisa/IIT SoftHand. In: 2017 IEEE World Haptics Conference (WHC). Munich: IEEE; 2017. p. 7-12.

23. Rossi M, Bianchi M, Battaglia E, Catalano MG, Bicchi A. HapPro: a wearable haptic device for proprioceptive feedback. IEEE Trans Biomed Eng. 2019; 66(1):138-49.

24. Patterson PE, Katz JA. Design and evaluation of a sensory feedback system that provides grasping pressure in a myoelectric hand. J Rehabil Res Dev. 1992;29(1):1-8.

25. Meek SG, Jacobsen SC, Goulding PP. Extended physiologic taction: design and evaluation of a proportional force feedback system. J Rehabil Res Dev. 1989;26(3):53-62.

26. Antfolk C, Balkenius C, Lundborg G, Rosén B, Sebelius F. A tactile display system for hand prostheses to discriminate pressure and individual finger localization. J Med Biol Eng. 2010;30(6):355-60.

27. Schoepp KR, Dawson MR, Schofield JS, Carey JP, Hebert JS. Design and integration of an inexpensive wearable mechanotactile feedback system for myoelectric prostheses. IEEE J Transl Eng Heal Med. 2018;6: $1-11$

28. Raspopovic S, Capogrosso M, Petrini FM, Bonizzato M, Rigosa J, Di Pino G, et al. Restoring Natural Sensory Feedback in Real-Time Bidirectional Hand Prostheses. Sci Transl Med. 2014;6(222):222ra19.

29. Tan DW, Schiefer MA, Keith MW, Anderson JR, Tyler J, Tyler DJ. A neural interface provides long-term stable natural touch perception. Sci Transl Med. 2014;6(257):257ra138

30. Ortiz-Catalan M, Håkansson B, Brånemark R. An osseointegrated humanmachine gateway for long-term sensory feedback and motor control of artificial limbs. Sci Transl Med. 2014;6(257):257re6. 
31. Schiefer MA, Graczyk EL, Sidik SM, Tan DW, Tyler DJ. Artificial tactile and proprioceptive feedback improves performance and confidence on object identification tasks. PLoS One. 2018;13(12):e0207659.

32. Clemente F, Valle G, Controzzi M, Strauss I, Iberite F, Stieglitz T, et al. Intraneural sensory feedback restores grip force control and motor coordination while using a prosthetic hand. J Neural Eng. 2019;16(2):026034.

33. Page DM, George JA, Kluger DT, Duncan C, Wendelken S, Davis T, et al. Motor control and sensory feedback enhance prosthesis embodiment and reduce phantom pain after long-term hand amputation. Front Hum Neurosci. 2018;12:1-16

34. Tabot GA, Dammann JF, Berg JA, Tenore FV, Boback JL, Vogelstein RJ, et al. Restoring the sense of touch with a prosthetic hand through a brain interface. Proc Natl Acad Sci. 2013;110(45):18279-84.

35. Stepp CE, An Q, Matsuoka Y. Repeated training with augmentative vibrotactile feedback increases object manipulation performance. PLoS One. 2012;7(2):e32743.

36. Witteveen HJB, Rietman HS, Veltink PH. Vibrotactile grasping force and hand aperture feedback for myoelectric forearm prosthesis users. Prosthetics Orthot Int. 2015;39(3):204-12.

37. Walker J, Blank A, Shewokis P, O'Malley M. Tactile Feedback of Object Slip Facilitates Virtual Object Manipulation. IEEE Trans Haptics. 2015;W(c):1.

38. Valle G, Petrini FM, Strauss I, Iberite F, D'Anna E, Granata G, et al. Comparison of linear frequency and amplitude modulation for intraneural sensory feedback in bidirectional hand prostheses. Sci Rep. 2018;8(1):16666

39. Clemente F, D'Alonzo M, Controzzi M, Edin BB, Cipriani C. Non-invasive, temporally discrete feedback of object contact and release improves grasp control of closed-loop myoelectric transradial prostheses. IEEE Trans Neural Syst Rehabil Eng. 2016;24(12):1314-22.

40. Markovic M, Schweisfurth MA, Engels LF, Bentz T, Wüstefeld D, Farina D, et al. The clinical relevance of advanced artificial feedback in the control of a multi-functional myoelectric prosthesis. J Neuroeng Rehabil. 2018;15(1):28.

41. Shehata AW, Engels LF, Controzzi M, Cipriani C, Scheme EJ, Sensinger JW. Improving internal model strength and performance of prosthetic hands using augmented feedback. J Neuroeng Rehabil. 2018;15(1):70.

42. Valle G, Mazzoni A, Iberite F, D'Anna E, Strauss I, Granata G, et al. Biomimetic Intraneural Sensory Feedback Enhances Sensation Naturalness, Tactile Sensitivity, and Manual Dexterity in a Bidirectional Prosthesis. Neuron. 2018; 100(1):37-45.e7.

43. Aboseria M, Clemente F, Engels LF, Cipriani C. Discrete Vibro-tactile feedback prevents object slippage in hand prostheses more intuitively than other modalities. IEEE Trans Neural Syst Rehabil Eng. 2018;26(8):1577-84.

44. Schiefer M, Tan D, Sidek SM, Tyler DJ. Sensory feedback by peripheral nerve stimulation improves task performance in individuals with upper limb loss using a myoelectric prosthesis. J Neural Eng. 2015;13(1):16001.

45. Raveh E, Friedman J, Portnoy S. Visuomotor behaviors and performance in a dual-task paradigm with and without vibrotactile feedback when using a myoelectric controlled hand. Assist Technol. 2018;30(5):274-80.

46. Markovic M, Schweisfurth MA, Engels LF, Farina D, Dosen S. Myocontrol is closed-loop control: incidental feedback is sufficient for scaling the prosthesis force in routine grasping. J Neuroeng Rehabil. 2018;15(1):81.

47. Schweisfurth MA, Markovic M, Dosen S, Teich F, Graimann B, Farina D. Electrotactile EMG feedback improves the control of prosthesis grasping force. J Neural Eng. 2016 Oct;13(5):056010.

48. Ninu A, Dosen S, Muceli S, Rattay F, Dietl H, Farina D. Closed-loop control of grasping with a myoelectric hand prosthesis: which are the relevant feedback variables for force control? IEEE Trans Neural Syst Rehabil Eng. 2014;22(5):1041-52.

49. Dosen S, Markovic M, Hartmann C, Farina D. Sensory feedback in prosthetics: a standardized test bench for closed-loop control. IEEE Trans Neural Syst Rehabil Eng. 2015;23(2):267-76.

50. Kingdom FAA, Prins N. Psychophysics: a practical introduction. San Diego: Academic; 2010

51. Berniker M, Kording K. Estimating the sources of motor errors for adaptation and generalization. Nat Neurosci. 2011;11:1454-61.

52. Wolpert DM, UKPMC Funders Group. Probabilistic models in human sensorimotor control. Hum Mov Sci. 2009:26(4):511-24.

53. Ernst MO, Banks MS. Humans integrate visual and haptic information in a statistically optimal fashion. Nature. 2002;415(6870):429-33.

54. Pylatiuk C, Kargov A, Schulz S. Design and evaluation of a low-cost force feedback system for myoelectric prosthetic hands. J Prosthet Orthot. 2006; 18(2):57-61.
55. Witteveen HJB, Luft F, Rietman JS, Veltink PH. Stiffness feedback for myoelectric forearm prostheses using vibrotactile stimulation. IEEE Trans Neural Syst Rehabil Eng. 2014;22(1):53-61.

56. Marcus PL, Fuglevand AJ. Perception of electrical and mechanical stimulation of the skin: implications for electrotactile feedback. J Neural Eng. 2009:6(6):066008.

57. Schweisfurth MA, Markovic M, Dosen S, Teich F, Graimann B, Farina D. Electrotactile EMG feedback improves the control of prosthesis grasping force. J Neural Eng. 2016;13(5):1-16.

58. Dosen S, Markovic M, Somer K, Graimann B, Farina D. EMG biofeedback for online predictive control of grasping force in a myoelectric prosthesis. J Neuroeng Rehabil. 2015;12(1):55.

\section{Publisher's Note}

Springer Nature remains neutral with regard to jurisdictional claims in published maps and institutional affiliations.
Ready to submit your research? Choose BMC and benefit from:

- fast, convenient online submission

- thorough peer review by experienced researchers in your field

- rapid publication on acceptance

- support for research data, including large and complex data types

- gold Open Access which fosters wider collaboration and increased citations

- maximum visibility for your research: over $100 \mathrm{M}$ website views per year

At $\mathrm{BMC}$, research is always in progress.

Learn more biomedcentral.com/submissions 